Canad. Math. Bull. Vol. 20 (2). 1977

\title{
SINGULAR ISOMETRIES IN ORTHOGONAL GROUPS
}

\author{
BY \\ GEORG GUNTHER
}

In this paper, we study the behaviour of singular isometries in orthogonal groups. These are isometries whose path is a singular subspace. We shall prove that the path of such a singular isometry is always even-dimensional. We shall use this result to show that the subgroup of the orthogonal group $O_{n}(K, Q)$ which is generated by the singular isometries is the commutator subgroup $\Omega_{n}(K, Q)$. In particular, in the case that $n=4$ and index $Q=2$, we shall obtain a nice geometric interpretation for the well-known result that $P \Omega_{n}(K, Q)$ is isomorphic to $P S L_{2}(K) \times P S L_{2}(K)$. In addition, we shall discuss some subgroups of the commutator group.

1. Introduction. Let $(V, Q)$ be an $n$-dimensional metric vector space over a field $K$, with a quadratic form $Q$. Let $f$ be the bilinear form associated with $Q$. The form $f$ induces an orthogonality on $V$. We say that a subspace $H$ is regular if $\operatorname{Rad} H=H^{\cap} H^{\perp}=0$ and $H$ is isotropic if $\operatorname{Rad} H=H$. A subspace $H$ is singular if $Q(h)=0$ for all $h \in H$. The index of $Q$ is the dimension of the maximal singular subspaces of $V$.

In this paper, we shall assume that $V$ is regular, that $K \neq G F(2)$, and that the index of $Q$ is at least 2. We use the notation of Ellers ([5], [6] and [7]) throughout this text.

A transformation $\pi$ of $V$ is called an isometry if it preserves $Q$ (and hence preserves $f$ ). The set of all isometries is the orthogonal group $O_{n}(K, Q)$. With every isometry we can associate two subspaces:

and

$$
\text { the path of } \pi:=B(\pi)=\{\pi(x)-x \mid x \in V\}
$$

$$
\text { the fix of } \pi:=F(\pi)=\{x \in V \mid \pi(x)=x\} .
$$

Clearly, $\operatorname{dim} B(\pi)+\operatorname{dim} F(\pi)=n$, and $B(\pi)^{\perp}=F(\pi)$. An isometry $\sigma$ is simple if $\operatorname{dim} B(\sigma)=1$; these simple isometries generate the group $O_{n}(K, Q)$. We say that an isometry $\pi$ is singular if $B(\pi)$ is a singular subspace; otherwise, we call $\pi$ non-singular.

We let $l(\pi)$ (the length of $\pi$ ) be the minimum number of simple isometries whose product yields $\pi$. If $l(\pi)=2$, we call $\pi$ a rotation. We shall use projective

Received by the editors March 24, 1976.

I wish to thank the geometers at the Technische Hochschule Darmstadt, where this paper was written in the summer of 1975 . 
language, and say "point", respectively "line" for a one, respectively twodimensional subspace. A line can then contain 0,1 , or 2 singular points, or consist entirely of singular points. We call such lines respectively elliptic, parabolic, hyperbolic, or singular, and say that a rotation is elliptic, parabolic, hyperbolic, or singular as its path is a line of the corresponding type. In the following theorem, we gather some well-known results.

THEOREM 1.1

(a) Let $\pi$ be an isometry, and $\sigma$ be a simple isometry. Then $B(\pi \sigma)=$ $B(\pi)+B(\sigma)$ if and only if $B(\sigma) \not \subset B(\pi)$, and $\operatorname{dim} B(\pi \sigma)=\operatorname{dim} B(\pi)-1$ if and only if $B(\sigma) \subset B(\pi)$.

(b) If $\pi$ is non-singular, then $l(\pi)=\operatorname{dim} B(\pi)$.

(c) If $\pi$ is singular, then $l(\pi)=\operatorname{dim} B(\pi)+2$.

(d) If $\sigma$ is simple and $B(\sigma)=K p$, then

$$
\sigma(x)=x-\frac{f(x, p)}{Q(p)} p .
$$

(e) If $\rho$ is a parabolic rotation with $B(\rho)=\langle s, z\rangle$ with $Q(s)=f(s, z)=0$ and $Q(z)=\lambda \neq 0$, then

$$
\rho(x)=x+\frac{\delta}{\lambda}[f(x, s) z-f(x, z+\delta s) s] \text { for some } \delta \in K .
$$

(f) If $\rho$ is a hyperbolic rotation, $B(\rho)=\langle s, t\rangle$ with $Q(s)=Q(t)=0$ and $f(s, t)=$ 1 , then $\rho(s)=\alpha s, \rho(t)=\alpha^{-1}$, for some $\alpha \in K$, and $\rho$ is uniquely determined by its effect on $s$.

The proof of these results will be omitted. See [4] for details; also [8] for a proof of (b) and (c).

If $H$ is any subspace, we let $O(H)=\left\{\pi \in O_{n}(K, Q) \mid B(\pi) \subset H\right\}$, and $O^{+}(H)=\{\pi \in O(H) \mid l(\pi)$ is even $\}$. In particular, if $H$ is a line, $O^{+}(H)$ is the group of all rotations whose path is the line $H$.

As in Ellers [5], we also define, for any isometry $\pi$, the set $P(\pi)$ by

$$
P(\pi):=\{x \in V \mid f(\pi(x)-x, x)=0\} .
$$

The condition " $x \in P(\pi)$ " is clearly equivalent to " $Q(\pi(x)-x)=0$ ", so that the size of $P(\pi)$ gives a measure of the occurrence of singular vectors in $B(\pi)$. Clearly, the isometry $\pi$ is singular if and only if $P(\pi)=V$.

2. Representation of singular isometries. In $1.1 \mathrm{~d}$, we see that a simple isometry can never be singular. This raises the question: for what singular subspaces $H$ does there exist a singular isometry whose path is $H$ ? Before answering this, we prove

Lemma 2.1. Let $A$ be a $k$-dimensional non-singular subspace containing a 
singular $k$-1-dimensional subspace $B$. The one of two cases occurs:

(a) $B \subset \operatorname{rad} A$, and all singular points of $A$ lie in $B$.

(b) $B \not \subset \operatorname{rad} A$, and $A$ contains a second singular $k$-1-dimensional subspace $C \neq B$.

Every singular point of $A$ lies in either $A$ or $B$. If $\sigma$ is any simple isometry such that $B(\sigma) \subset A$, then $\sigma(B)=C$.

\section{Proof}

(a) This is immediate, as otherwise $A$ would be singular.

(b) Now $B \not \subset \operatorname{rad} A$. Let $a$ be a non-singular vector of $A$, and $b$ a vector of $B$ such that $f(a, b) \neq 0$. Then $\langle a, b\rangle$ is a hyperbolic line, and hence contains a second singular vector $c \notin B$. Then $K c \oplus\left(c^{\perp} \cap B\right)=C$ is a second singular $k$-1-dimensional subspace of $A$. Now suppose that $x=b+\alpha c$ with $b \in B$ is any vector of $A$. Then $Q(b+\alpha c)=\alpha f(b, c)=0$ if and only if $\alpha=0$ and $x \in B$, or $f(b, c)=0$ and $x \in C$. Finally, let $B(\sigma)=K a \subset A$. Then there exists a hyperbolic line $\left\langle b^{\prime}, c^{\prime}\right\rangle$ with $b^{\prime} \in B$ and $c^{\prime} \in C$ containing $a$ such that $\sigma\left(\left\langle b^{\prime}\right\rangle\right)=\left\langle c^{\prime}\right\rangle$. Hence $\sigma(B)=C$.

This enables us to prove

LEMMA 2.2. Let $\pi$ be singular and $\sigma$ be simple. Then $\pi \sigma$ is non-singular.

Proof. Since $\pi$ is singular, we know that $P(\pi)=V$, and hence $f(\pi \sigma(x), \sigma(x))=f(x, x)$ for all $x \in V$. If $\pi \sigma$ were also singular, we would also have $f(\pi \sigma(x), x)=f(x, x)$ for all $x \in V$, implying that $f(\pi \sigma(x), B(\sigma))=0$ for all $x \in V$. But then $B(\sigma) \subset \operatorname{rad} V$, contrary to the assumption that $V$ is regular.

We are now able to answer the question posed at the top in

THEOREM 2.3. Let $\pi$ be a singular isometry. Then $\operatorname{dim} B(\pi)$ is even.

Proof. Suppose $l(\pi)=k+2$. Let $K p$ be a non-singular point such that $p \notin B(\pi)^{\perp}$, and let $B(\sigma)=K p$. By 2.2 , we can write $\pi=\rho \sigma$, where $\rho$ is a non-singular isometry of length $k+1$. Clearly, $B(\pi), B(\sigma) \subset B(\rho)=A$, and by our choice of $p$, we ensure that $B(\pi) \not \subset \operatorname{rad} A$. Thus Theorem 2.1 pertains. Now $\pi(B(\pi))=B(\pi)$. But if $l(\pi)$ were odd, then $\pi(B(\pi))$ would be the image of $B(\pi)$ under an odd number of simple isometries, which is distinct from $B(\pi)$ by 2.1 . Hence $l(\pi)=k+2$ is even, and thus $\operatorname{dim} B(\pi)=k$ is even.

We observe that if $\pi$ is a singular isometry, then $B(\pi)$ is singular, and hence isotropic, implying that $B(\pi) \subset F(\pi)$. If char $K=p$, then let $\pi$ be any singular isometry. Then

$$
\begin{aligned}
\pi^{p}(x)-x & =\pi^{p}(x)-\pi^{p-1}(x)+\pi^{p-1}(x)-\cdots+\pi(x)-x \\
& =\sum_{i=1}^{p} \pi^{i-1}(\pi(x)-x)=\sum_{i=1}^{p}(\pi(x)-x)=0 .
\end{aligned}
$$


Thus we have proved

LemMA 2.4. If Char $K=p$, and $\pi$ is singular, then $\pi^{p}=1$.

We now state the representation theorem for singular isometries.

Theorem 2.5. Let $\pi$ be an isometry. Then $\pi$ is singular if and only if

$$
\pi(x)=x+\sum_{i, j=1}^{k} \alpha_{i j} f\left(x, s_{j}\right) s_{i}
$$

where the matrix $\left(\alpha_{i j}\right)$ is skew-symmetric with zeros down the main diagonal and $S=\left\langle s_{1}, \ldots, s_{k}\right\rangle$ is a $k$-dimensional singular subspace.

The proof of this theorem involves straightforward calculation, and thus is omitted. Now, if $H$ is any singular subspace, we let $O(H)$ be the group generated by all singular isometries whose path lies in $H$. As corollaries of 2.5 we have

COROllary 2.6. If $H$ is a singular subspace of even dimension, then there exists a singular isometry whose path is $H$.

Corollary 2.7. If $H$ is a singular line, then $O(H)$ is isomorphic to $(K,+)$. If $\pi \in O(H)$, then $\pi(x)=x+\alpha[f(x, s) t-f(x, t) s]$, where $\langle s, t\rangle=H$.

The singular rotations are important because they generate the singular isometries. This is the content of the next

THEOREM 2.8. Let $\pi$ be a singular isometry. Let $\operatorname{dim} B(\pi)=2 k$. Then $\pi$ is the product of $k$ singular rotations.

Proof. Choose $s \in B(\pi)$. Then there exists some $a \in V$ such that $\pi(a)=a+s$. But then $Q(\pi(a))=Q(a)=Q(a+s)=Q(a)+f(a, s)$, implying that $f(a, s)=0$. Clearly $B(\pi) \not \subset a^{\perp}$, and so we can find some $t \in B(\pi)$ for which $f(a, t)=1$. Let $\tau$ be the singular rotation given by $\tau(x)=x+f(x, s) t-f(x, t) s$. Then $a \in F(\tau \pi)$. Since $F(\pi) \subset F(\tau \pi)$, we thus see that $F(\pi)$ is properly contained in $F(\tau \pi)$. Since $\tau \pi$ is singular, we thus conclude that $\operatorname{dim} B(\tau \pi)=\operatorname{dim} B(\pi)-2$. The result now follows by induction.

In the next lemma, we study products of two singular or two parabolic rotations.

LEMMA 2.9

(a) Let $\tau_{1}, \tau_{2}$ be two singular rotations such that $B\left(\tau_{1}\right)=\langle s, t\rangle$ and $B\left(\tau_{2}\right)=$ $\langle s, r\rangle$. If $f(t, r)=0$, then the product $\tau_{1} \tau_{2}$ is a singular rotation. If $f(t, r) \neq 0$, the product is a parabolic rotation. In either case, we have $\tau_{1} \tau_{2}=\tau_{2} \tau_{1}$.

(b) Let $\rho$ be a parabolic rotation with $B(\rho)=\langle s, z\rangle$, such that $Q(s)=0=$ $f(s, z)$, and $Q(z) \neq 0$. Then $\rho$ is the product of two singular rotations $\tau_{1}, \tau_{2}$ such that $B\left(\tau_{1}\right) \cap B\left(\tau_{2}\right)=K s$.

(c) Let $\rho_{1}, \rho_{2}$ be two parabolic rotations with $B\left(\rho_{1}\right) \cap B\left(\rho_{2}\right)=K s$, and $Q(s)=$ 0 . Then $\rho_{1} \rho_{2}=\rho_{2} \rho_{1}$, and the product is either singular or parabolic. 
(d) Let $\tau$ be a singular rotation. Then $\tau=\rho_{1} \rho_{2}$, where the $\rho_{i}$ are parabolic rotations with $B\left(\rho_{1}\right) \cap B\left(\rho_{2}\right)=K s \subset B(\tau)$.

\section{Proof}

(a) If $\tau_{1} \neq \tau_{2}$, then by $1.1 \mathrm{~b}$, c, we have $1\left(\tau_{1} \tau_{2}\right)=2$. If $f(t, r)=0$, then $B\left(\tau_{1} \tau_{2}\right) \subset$ $\langle s, t, r\rangle$, which is a singular subspace, implying the result. We can assume that $B\left(\tau_{1}\right) \neq B\left(\tau_{2}\right)$, as otherwise 2.7 yields the result. But then $F\left(\tau_{1} \tau_{2}\right)=$ $\left\{x \in V \mid \tau_{1} \tau_{2}(x)=x\right\} \subset\left\{x \mid \tau_{2}(x)-x \in K s\right\}$. But $\tau_{2}(x)=x+\alpha s$ implies that $f(x, s)=$ 0 . Hence $F\left(\tau_{1} \tau_{2}\right) \subset s^{\perp}$, or $s \in B\left(\tau_{1} \tau_{2}\right)$. Hence $\tau_{1} \tau_{2}$ is a rotation fixing $s$. If $\tau_{1} \tau_{2} \neq$ id., then in view of $1.1 \mathrm{f}$, we can conclude that $\tau_{1} \tau_{2}$ is parabolic. A simple computation shows that $\tau_{1} \tau_{2}=\tau_{2} \tau_{1}$.

(b) In view of $1.1 \mathrm{e}$, we can write $\rho(x)=x+\delta / \lambda[f(x, s) z-f(x, z+\delta s) s]$, where $\lambda=Q(z)$. Choose a singular vector $t \in s^{\perp}$ such that $f(t, z)=1$, and let $\tau$ be the singular isometry $\tau(x)=x+\delta[f(x, t) s-f(x, s) t]$. Then a simple computation shows that the product $\tau \rho$ is again a singular rotation $\tau^{\prime}$ with path $B\left(\tau^{\prime}\right)=\langle s, \delta z-\delta \lambda t\rangle$.

(c) As in (a), we see that the product $\rho_{1} \rho_{2}$ is again a rotation whose path contains $K s$, and which fixes the vector $s$. Hence, either $\rho_{1} \rho_{2}=$ id, or, by $1.1 \mathrm{f}$, $\rho_{1} \rho_{2}$ is not hyperbolic. Again, a simple computation shows that $\rho_{1} \rho_{2}=\rho_{2} \rho_{1}$.

(d) As in (b), this again follows constructively.

3. The group of a singular point. We can now use Lemma 2.9 to define a certain family of subgroups of the group $O_{n}(K, Q)$. We let $K s$ be any singular point, and we define the set

$I(s)=\bigcup O^{+}(H)$, where this union is taken over all lines $H$ that contain the point $K s$. We also define a subset of $I(s)$ by $R(s)=\bigcup O^{+}(H)$, where the line $H$ contains the point $K s$ and is itself contained in the hyperplane $s^{\perp}$. We prove the following

THEOREM 3.1. The set $I(s)$ is a group. $R(s)$ is a normal abelian subgroup of $I(s)$, and the factor group $I(s) / R(s)$ is isomorphic to the multiplicative group of the field $K$.

Proof. Let $\tau \neq \mathrm{id} \neq \rho$ be elements of $I(s)$. Since $s \in B(\tau) \cap B(\rho)$, we see that $\operatorname{dim} B(\tau \rho) \leq 3$. Also, $\operatorname{dim} B(\tau \rho)$ is even, and hence the product $\tau \rho$ is a rotation or $\tau \rho=$ id. If $B(\tau)=B(\rho)$, there is nothing to prove. So assume that $B(\tau) \cap$ $B(\rho)=K s$. Then $F(\tau \rho)=\{x \in V \mid \tau \rho(x)=x\} \subset\{x \in V \mid \rho(x)-x \in K s\}$. But $\rho(x)=$ $x+\alpha s$ implies that $f(x, s)=0$, and thus $F(\tau \rho) \subset s^{\perp}$, or equivalently, $s \in B(\tau \rho)$. Thus $I(s)$ is a subgroup. The same reasoning shows that $R(s)$ is a subgroup which is clearly normal in $I(s)$. The elements of $R(s)$ are either singular or parabolic rotations, and so we see from 2.9 that the elements of $R(s)$ all commute. We now fix a hyperbolic line through $s$, and let $\tau$ be any hyperbolic rotation whose path is a second hyperbolic line. Then $\tau(s)=\alpha(s)$ for some 
$\alpha \in K$. We let $\tau^{\prime}$ be the hyperbolic rotation whose path is the given hyperbolic line $H$ such that $\tau^{\prime}(s)=\alpha^{-1} s$. Then $\tau \tau^{\prime}(s)=s$ and hence $\tau \tau^{\prime}$ is a parabolic or singular rotation. Also, the rotation $\tau$ uniquely determines the rotation $\tau^{\prime}$. We have thus shown that every rotation $\tau$ can be expressed as a product of a rotation in $\mathrm{O}^{+}(\mathrm{H})$ and a rotation in $R(s)$, and that this decomposition is unique. We define a map $\psi: I(s) \rightarrow O^{+}(H)$, where $\psi(\tau)$ is the uniquely determined element of $\mathrm{O}^{+}(H)$ in the decomposition described above. Clearly, $\psi(\mathrm{id})=\mathrm{id}$, and $\tau \tau^{\prime}=\psi(\tau) \rho \cdot \psi\left(\tau^{\prime}\right) \rho^{\prime}$, where $\rho, \rho^{\prime} \in R(s)=\psi(\tau) \psi\left(\tau^{\prime}\right)\left[\psi\left(\tau^{\prime}\right)^{-1} \rho \psi\left(\tau^{\prime}\right) \rho^{\prime}\right]$, so that $\psi\left(\tau \tau^{\prime}\right)=\psi(\tau) \psi\left(\tau^{\prime}\right)$, since $\psi\left(\tau^{\prime}\right)^{-1} \rho \psi\left(\tau^{\prime}\right) \rho^{\prime} \in R(s)$. Hence the map $\psi$ is a homomorphism whose kernel is clearly $R(s)$. Hence we have $I(s) / R(s)$ is isomorphic to $\mathrm{O}^{+}(H)$, where $H$ is a hyperbolic line, and from Dieudonné [4] we know that $\mathrm{O}^{+}(\mathrm{H})$ is isomorphic to the multiplicative group of the field.

If $\operatorname{dim} V=3$ and index $Q=1$, we have a nice geometric interpretation of the group $I(s)$, which is due to Dr. H. Mäurer at the Technische Hochschule Darmstadt. For now the quadric is a Möbius plane, if we define the cycles as the plane sections of $V$ with the quadric. Then the derived plane in the point $K s$ is an affine plane. If $H$ is a hyperbolic line through $K s$ which meets the quadric in a second point $K t$, then all the hyperbolic rotations with path $H$ induce central dilatations with center $K t$ in the derived affine plane. If $H$ is a parabolic line, on the other hand, then the corresponding parabolic rotations induce all the translations in a given direction. Thus $R(s)$ is isomorphic to the translation group of this affine plane, and this group is, as we know, an abelian group.

4. A class of subgroups of $O_{n}$. In this section we describe another class of subgroups of $O_{n}$. Before doing this, we require a general

LEMMA 4.1. Let $A$ be an n-2-dimensional subspace of an n-dimensional vector space. Define $T(A):=\left\langle\left\{\tau \in S L_{n}(K) \mid \tau\right.\right.$ is a transvection and $\left.\left.A \subset F(\tau)\right\}\right\rangle$, and $B T(A):=\langle\{\pi \in T(A) \mid B(\pi) \subset A\}\rangle$. Then $T(A), B T(A)$ are groups; $B T(A)$ is a normal subgroup of $T(A)$, and the factor group $T(A) / B T(A)$ is isomorphic to the group $S L_{2}(K)$.

Proof. Clearly, $T(A)$ and $B T(A)$ are subgroups, and $B T(A)$ is normal in $T(A)$. We now define a map $\delta$ as follows

$$
\delta:\left\{\begin{aligned}
T(A) & \rightarrow G L_{2}(K) \\
\pi & \rightarrow \delta(\pi)
\end{aligned}\right.
$$

where

$$
\delta(\pi):\left\{\begin{array}{c}
V / A \rightarrow V / A \\
x+A \rightarrow \pi(x)+A
\end{array}\right.
$$

This is a well-defined homomorphism, since $A \subset F(\pi)$ for all $\pi \in T(A)$. Now 
$\operatorname{ker} \delta=\{\pi \mid \pi(x)+A=x+A$ for all $x \in V\}=\{\pi \mid B(\pi) \subset A\}$. Hence $\operatorname{ker} \delta=$ $B T(A)$. Now choose any transvection $\tau \in T(A)$. We know that $\tau(x)=x+\psi(x) b$, where $A \subset \operatorname{ker} \psi$. Then $\delta(\tau)(x+A)=x+\psi(x) b+A=x+A+\psi(x+A)(b+A)$, and $\delta(\tau)(b+A)=b+A$. Thus we see that $\delta(\tau)$ is a transvection of $V / A$, and hence $\delta$ is a homomorphism into $S L_{2}(K)$. Now let $T$ be any transvection of $V / A$, say $T(x+A)=(x+A)+\phi(x+A)(c+A)$ with $c \notin A$. Then $A+K c$ is a hyperplane of $V$, and thus the map $\tau^{\prime}(x)=x+\phi^{\prime}(x) c$ is a transvection in $T(A)$, where $H=\operatorname{ker} \phi^{\prime}$ and $\phi^{\prime}(y)=1=\phi(y+A)$ for a suitable $y \notin A+K c$. Then we see at once that $\delta\left(\tau^{\prime}\right)=T$. Hence $\delta$ is surjective. Thus the theorem is proved.

We now return to the study of the orthogonal group, and use Theorem 4.1 to prove

THeOREM 4.2. Let $H$ be a fixed singular line. Let $L(H):=\left\{H^{\prime} \mid \operatorname{dim} H^{\prime}=2\right.$ and $H \cap H^{\prime} \neq 0$, and $H^{\prime}$ singular $\}$. Define $G(H):=\left\langle\left\{\pi \in O_{n} \mid B(\pi) \in L(H)\right\}\right\rangle$. Then $O(H)$ is a normal subgroup of $G(H)$, and the factor group $G(H) / O(H)$ is isomorphic to the group $T\left(H^{\perp} / H\right)$, which is defined as in the statement of Theorem 4.1.

Proof. We first observe that if $\tau$ is any singular rotation whose path is a line of $L(H)$, then $\tau$ fixes $H$. This is a direct consequence of $2.1 \mathrm{~b}$. As in the proof of Theorem 4.1, we define a map $\rho$ as follows

$$
\rho:\left\{\begin{aligned}
G(H) & \rightarrow G L_{n-2}(V / H) \\
\pi & \rightarrow \rho(\pi)
\end{aligned}\right.
$$

where $\rho(\pi)$ is defined by

$$
\rho(\pi):\left\{\begin{aligned}
V / H & \rightarrow V / H \\
x+H & \rightarrow \pi(x)+H
\end{aligned}\right.
$$

Now we argue as in 4.1. We see that $\rho$ is a homomorphism with kernel $O(H)$. If $\tau$ is any singular rotation with path $a$ line in $L(H)$, then as in 4.1, we see that $\rho(\tau)$ is a transvection of $V / H$. Indeed, if $H \cap B(\tau)=K s$, then $\rho(\tau)$ is a transvection whose path is $K(t+H)$, where $t$ is a second vector of $B(\tau)$, and whose fix is the hyperplane $s^{\perp} / H$ of $V / H$. But $s \in H$ implies that $H^{\perp} \subset s^{\perp}$, and thus $\rho(\tau)$ is a transvection of $T\left(H^{\perp} / H\right)$. Thus $\rho$ is a homomorphism into $T\left(H^{\perp} / H\right)$. Now consider any $s \in H$, and any non-singular $z \in s^{\perp}$. Then $\langle s, z\rangle$ is a parabolic line, and let $\sigma$ be a parabolic rotation with this line as path. Then by $2.9 \mathrm{~b}$, we know that $\sigma$ can be written as the product of two singular rotations which are clearly elements of $G(H)$. Hence $\sigma \in G(H)$. But then we can check that $\rho(\tau)$ is a transvection of $V / H$ whose path is $K(z+H)$, and whose fix is $s^{\perp} / H$. Thus these parabolic rotations yield all the transvections of $T\left(H^{\perp} / H\right)$ whose path is a non-singular point. Conversely, if $T$ is any transvection in $T\left(H^{\perp} / H\right)$, then $T$ is induced by a singular or a parabolic rotation in $G(H)$, 
depending whether $B(T)$ is a singular or non-singular point. Thus $\rho$ is surjective, and the theorem is proved. The following corollary is of particular interest.

Corollary 4.3. If $\operatorname{dim} V=4$, then $G(H) / O(H)$ is isomorphic to $S L_{2}(K)$.

Proof. In this case, $H=H^{\perp}$, and hence $H^{\perp} / H=0$. But then 4.1 allows us to deduce that $B T\left(H^{\perp} / H\right)=1$, and hence $T\left(H^{\perp} / H\right) / B T\left(H^{\perp} / H\right)$ is isomorphic to $T\left(H^{\perp} / H\right)$, which in turn is isomorphic to $S L_{2}(K)$.

5. The group generated by singular isometries. We now let $S$ be the set of all singular isometries, and $G(S)$ be the subgroup of $O_{n}$ which is generated by the elements of $S$. We let $\Omega_{n}$ denote the commutator subgroup of $O_{n}(K, Q)$, and let $O_{n}^{+}(K, Q)$ be the subgroup of $O_{n}(K, Q)$ which is generated by the rotations. As in [4], we let $\theta$ be the spinor norm, which is the map $\theta: O_{n}^{+}(K, Q) \rightarrow K^{*} / K^{*^{2}}$. The spinor norm is defined as follows: suppose $\pi \epsilon$ $O_{n}^{+}(k, Q)$, and $\pi=\sigma_{1} \cdots \sigma_{k}$, where the $\sigma_{i}$ are simple isometries and $B\left(\sigma_{i}\right)=$ $K p_{i}$. Then $\theta(\pi)=Q\left(p_{1}\right) \cdots Q\left(p_{k}\right) \cdot K^{*^{2}}$. From [4], we know that $\theta$ is a surjective homomorphism whose kernel is $\Omega_{n}(K, Q)$, and hence $O_{n}^{+}(K, Q) / \Omega_{n}(K, Q)$ is isomorphic to $K^{*} / K^{* 2}$.

Now let $\rho$ be any parabolic rotation with path $\langle s, z\rangle$ where $Q(s)=f(s, z)=0$, and $Q(z) \neq 0$. Then any other vector in $\langle s, z\rangle$ can be expressed in the form $x=\alpha(\beta s+z)$, and so $Q(x)=\alpha^{2} Q(z)$. But $\rho$ is a product of two simple isometries whose path lies in $\langle s, z\rangle$ and thus $\theta(\rho)=\alpha^{2} \beta^{2} Q(z)^{2} K^{* 2}=K^{* 2}$, implying that the parabolic rotations all lie in $\Omega_{n}(K, Q)$. But by $2.9 \mathrm{~d}$, we see that every singular rotation can be expressed as the product of two parabolic rotations. Thus we have proved

LEMMA 5.1. The group $G(S)$ is a subgroup of $\Omega_{n}(K, Q)$.

We can improve upon this lemma. We do this in the next two theorems.

THEOREM 5.2. If $n \geq 5$, then $G(S)=\Omega_{n}(K, Q)$.

Proof. If char $K=2$, we know from [4] that $\Omega_{n}(K, Q)$ is a simple group for $n \geq 5$. Since $G(S)$ is clearly a normal subgroup of $\Omega_{n}(K, S)$, we therefore conclude the result. If char $K \neq 2$, we need only check that $G(S)$ is not contained in the center $Z_{n}$ of $O_{n}(K, Q)$. But $Z_{n}=\{1,-1\}$, and clearly no singular rotation lies in $Z_{n}$. Hence we may apply Theorem 5.27 of Artin [1], to conclude that $G(S)$ contains the group $\Omega_{n}(K, Q)$. This again proves the result.

We deal separately with the case that $n=4$ and index $Q=2$. In this case the quadric is a hyperboloid and contains two families of singular lines. We denote these two families $L_{1}$ and $L_{2}$. Then any two distinct lines of $L_{1}$, respectively $L_{2}$, span the whole space, and every line of $L_{1}$ meets every line of $L_{2}$. Also, every singular point lies on exactly one line of each family. We define $G\left(L_{1}\right)$, 
respectively $G\left(L_{2}\right)$, to be the group generated by all the singular rotations whose path lies in $L_{1}$, respectively in $L_{2}$. Let $\tau_{1}$ be a singular rotation in $G\left(L_{1}\right)$, and $\tau_{2}$ be a singular rotation whose path lies in $L_{2}$. In view of $2.9 \mathrm{a}$, we know that $\tau_{1}$ and $\tau_{2}$ commute. Hence we have proved

Lemma 5.3. Let $\pi_{1} \in G\left(L_{1}\right)$, and $\pi_{2} \in G\left(L_{2}\right)$. Then $\pi_{1} \pi_{2}=\pi_{2} \pi_{1}$.

We now strengthen this result in

Lemma 5.4. Suppose that $\pi \in G\left(L_{1}\right)$, and that $\pi$ fixes all the lines in $L_{1}$. Then $\pi \in Z_{4}=$ center of the group $\mathrm{O}_{4}(K, Q)$.

Proof. By 5.3, we know that $\pi$ fixes all the lines in $L_{2}$. If $\pi$ also fixes all the lines of $L_{1}$ then $\pi$ fixes all the point of the quadric, since every singular point lies on two fixed lines. But then $\pi$ is a homothety (see [1]). Since $\pi$ is also an isometry, we conclude that $\pi=1$ or -1 .

We now check that $-1 \in G\left(L_{1}\right)$. For this purpose we decompose $V$ into two hyperbolic planes $V=\left\langle s_{1}, s_{2}\right\rangle(1)\left\langle s_{3}, s_{4}\right\rangle$. Then we define the following three singular isometries: (we assume char $K \neq 2$, as otherwise the result is trivial)

$$
\begin{aligned}
& \pi_{1}(x)=x+2\left[f\left(x, s_{3}\right) s_{1}-f\left(x, s_{1}\right) s_{3}\right] \\
& \pi_{2}(x)=x+2\left[f\left(x, s_{4}\right) s_{2}-f\left(x, s_{2}\right) s_{4}\right] \\
& \pi_{3}(x)=x+2\left[f\left(x, s_{1}+s_{4}\right)\left(s_{2}-s_{3}\right)-f\left(x, s_{2}-s_{3}\right)\left(s_{1}+s_{4}\right)\right]
\end{aligned}
$$

These are three singular rotations, and it is easy to see that they belong to $G\left(L_{1}\right)$. Now an easy, though laborious, calculation shows that the product $\pi_{1} \pi_{2} \pi_{3}=-1$.

We now choose a particular line $H$ in $L_{2}$. Recalling the definition of the group $G(H)$ (Theorem 4.2), we see at once from the preceding results that $G(H)$ is isomorphic to the direct product of $G\left(L_{1}\right)$ with $O(H)$. From this we deduce at once that the factor group $G(H) / O(H)$ is isomorphic to the group $G\left(L_{1}\right)$. Combining this with Corollary 4.3 , we see that the group $G\left(L_{1}\right)$ (and hence $\left.G\left(L_{2}\right)\right)$ is isomorphic to the group $S_{2}(K)$. Thus we have proved

THEOREM 5.5. The group $G(S)$ is isomorphic to $G\left(L_{1}\right) \times G\left(L_{2}\right)$ if char $K=2$. If char $K \neq 2$, then $G(S) / Z_{4}$ is isomorphic to $G\left(L_{1}\right) / Z_{4} \times G\left(L_{2}\right) / Z_{4}$. The groups $G\left(L_{i}\right)$ are isomorphic to $S L_{2}(K)$.

Thus we see that the group $G(S)$ is always the commutator subgroup $\Omega_{n}(K, Q)$, (see Dieudonne [4]). Thus we see why the commutator displays the uncharacteristic behaviour of being the direct product of two groups in the case that $\operatorname{dim} V=4$. This is a consequence of the geometric result that a hyperboloid is a ruled quadric, admitting the two families of lines. 


\title{
BIBLIOGRAPHY
}

1. E. Artin, Geometric Algebra, Interscience Tracts No. 3, New York-London, Interscience Publications, 1957.

2. R. Baer, Linear Algebra and Projective Geometry, New York, Academic Press, 1952.

3. J. Dieudonné, Sur les générateurs des groupes classiques, Summa Bras. Math. 3 (1955), pp. 149-178.

4. J. Dieudonné, La géométrie des groupes classiques, (Trois. Ed.) Ergeb. d. Math. 5; Springer Verlag, 1971.

5. E. W. Ellers, Length of a unitary transformation of characteristic 2, J. reine angew. Math. 281 (1976) pp. 6-12.

6. E. W. Ellers, The length of a unitary transformation, J. reine angew. 281 (1976) pp. 1-5.

7. E. W. Ellers and R. Patton, Zur geschichte der Erzeugnis von Bewegungsgruppen, Mitteil. Math. Ges. Hamburg, X, 2, 1973.

8. B. Huppert, Geometric Algebra, Lecture Notes, University of Illinois at Chicago Circle, 1970.

9. K-H. Meyer, Transvektionsrelationen in metrischen Vektorräumen der Charakteristik 2, J. reine angew. Math. 233 (1968), 189-199.

10. P. Scherk, On the decomposition of orthogonalities into symmetries, Proc. Amer. Soc. 1 (1950), 481-491.

\author{
SCARborough College, \\ UNIVERSITY OF TORONTO, \\ Toronto, Ontario, Canada
}

